\title{
BMI open Alcohol involvement in aggression between intimate partners in New Zealand: a national cross-sectional study
}

\author{
Jennie L Connor, ${ }^{1}$ Kypros Kypri, ${ }^{1,2}$ Melanie L Bell, ${ }^{1}$ Kimberly Cousins ${ }^{1}$
}

To cite: Connor JL, Kypri K, Bell ML, et al. Alcohol involvement in aggression between intimate partners in New Zealand: a national cross-sectional study. BMJ Open 2011;1:e000065. doi:10.1136/bmjopen2011-000065

- Prepublication history for this paper is available online. To view these files please visit the journal online (http://bmjopen.bmj.com)

Received 17 January 2011 Accepted 7 June 2011

This final article is available for use under the terms of the Creative Commons Attribution Non-Commercial 2.0 Licence; see http://bmjopen.bmj.com

${ }^{1}$ Department of Preventive and Social Medicine, University of Otago, Dunedin, New Zealand

${ }^{2}$ Centre for Clinical

Epidemiology \& Biostatistics, School of Medicine and Public Health, University of Newcastle, Newcastle, Australia

Correspondence to Professor Jennie Connor; jennie.connor@otago.ac.nz

\section{ABSTRACT}

Objectives: To examine the role of alcohol at the time of aggressive incidents between intimate partners in the general population by gender, by estimating (1) prevalence and severity of aggression, and drinking at the time, (2) associations of drinking at the time of the aggression with reported severity, anger and fear, and (3) association of usual drinking patterns with partner aggression.

Design: A national survey of 18-70-year-olds using an electoral roll sample obtained self-reported alcohol consumption, partner's alcohol consumption and details of the most severe partner aggression by the respondent and towards the respondent in the past 2 years. The mean scores for associated severity, anger and fear were analysed by gender and alcohol involvement. Multinomial models estimated associations of drinking patterns with aggression to and from the respondent.

Results: The response rate was $49 \%(n=1925)$. Men and women reported similar prevalence of victimisation and perpetration of aggression $(11-15 \%)$. Alcohol was involved in more than $25 \%$ of incidents, and reported more by women than by men, particularly male-only drinking when the respondent was the victim. Women reported greater severity, anger and fear with victimisation than men, and drinking was associated with greater reported severity. Heavy episodic drinking by respondents was associated with a threefold increase in victimisation and doubling of perpetration of aggression involving alcohol. Heavy episodic drinking by either partner was also associated with drinking being involved in reported aggression.

Conclusions: The experience of intimate-partner aggression in a cross-section of households differs by gender and the involvement of alcohol, and 'counts' of aggressive acts in a population-based survey do not reflect the reality of gender differences. Heavy episodic drinking patterns are associated with more aggression involving alcohol within relationships, and alcohol involvement is associated with increased severity.

\section{INTRODUCTION}

The role of alcohol in interpersonal violence has been consistently identified in a range of contexts internationally, with the strength of

\section{ARTICLE SUMMARY}

\section{Article focus}

- The gendered nature of partner aggression and the escalation of aggression by the involvement of alcohol are important considerations in prevention of domestic violence.

- Heavy drinking in intimate partnerships is associated with more partner violence, but the role of alcohol at the time of aggression and gender differences in the experience of partner aggression are not well understood.

\section{Key messages}

- Men and women report similar numbers of aggressive acts involving partners, but women report greater severity, anger and fear associated with these.

- Drinking by one or both partners increases levels of severity, anger and fear reported by victims.

- Heavy episodic drinking is associated with increased victimisation as well as perpetration of partner aggression involving alcohol.

Strengths and limitations of this study

- The study involved an unselected general population sample, and included aggressive acts of all levels of severity.

- The modest response rate means the prevalence of aggression and alcohol involvement are likely to have been underestimated owing to selection biases, which may differ by gender, and study power is limited.

- Information biases may have resulted from the sensitivity of the questions, differences in reporting by gender and the reporting of partners' drinking by respondents rather than the partners themselves.

the association being culturally dependent. ${ }^{1}$ In New Zealand (NZ), a recent populationbased survey reported that half of all physical and sexual assaults involved a perpetrator who had been drinking, and recent police data suggest that in one-third of violence offences, the offender had been drinking. ${ }^{2}$ The most serious offences dealt with by police involve higher proportions of alcohol involvement, up to $50 \%$ in cases where force 
was required to be used by police. ${ }^{3}$ Among self-reported assaults involving a perpetrator who had been drinking, $15 \%$ of physical assaults and $22 \%$ of sexual assaults were by an intimate partner. ${ }^{2}$

Higher rates of aggression have been consistently reported among intimate partners who are heavy drinkers, with alcohol use increasing the occurrence and severity of incidents, ${ }^{4-6}$ but the use of alcohol by partners at the time of incidents of intimate-partner aggression has only recently been the subject of systematic investigation, and would be expected to vary by country and by gender.

There are a number of reasons for expecting that women's experiences of partner aggression, and particularly aggression involving alcohol, would be different from men's. When aggression involves alcohol, there is quite often drinking by both parties, but it has been observed that drinking by the victim is less likely if the victim is female, ${ }^{7}$ and incidents involving drinking by only the female partner are uncommon. ${ }^{8}$ As well as this, there is evidence suggesting that alcohol consumption increases aggressiveness more for men than for women, ${ }^{9}$ that men generally drink more heavily than women, ${ }^{10}$ and that men experience a great deal more violence in other settings than women do. ${ }^{11}$

Measures of partner aggression, and the role of alcohol in such incidents, that are suitable for use in population surveys are still being developed and refined. The use of the Conflict Tactics Scale, ${ }^{12}$ while common, has been subject to considerable criticism. ${ }^{4}{ }^{13}{ }^{14}$ In particular, it appears to minimise gender differences in violence, focusing on frequency of incidents of aggression and failing to capture gender differences in the severity and dynamics of those incidents. Studies therefore tend to find little difference in the experience of partner aggression between men and women. In response to these shortcomings, a method was developed for the Gender, Alcohol, and Culture International Study (GENACIS) surveys, ${ }^{4}$ grounded in previous research ${ }^{15}{ }^{16}$ which asks the respondent to identify 'the most aggressive thing that has been done to you' and then asks for details about the chosen incident. A parallel set of questions can then be asked about perpetration of aggression towards a partner.

We applied this instrument in a population survey in $\mathrm{NZ}$ in order to describe the range of experiences of men and women with aggression in intimate partnerships, and the involvement of alcohol at the time of aggressive incidents.

\section{AIMS OF THIS STUDY}

To describe, in a general population sample:

1. the prevalence and severity of aggressive incidents between partners, and proportion that involve drinking by one or both partners, by gender;

2. the associations between gender, alcohol involvement and reported severity of aggression, anger and fear;

3 . the association of usual drinking pattern with the occurrence of partner aggression.

\section{METHODS}

\section{Design}

The study had a cross-sectional design, using selfreported data on alcohol consumption and incidents of aggression involving intimate partners.

In 2007, we conducted a national survey of NZ adults. A simple random sample of $4000 \mathrm{NZ}$ residents, aged 18-70, was drawn from the combined electoral roll, a national voter registration system covering more than $90 \%$ of residents 18 years and older. Following an invitation letter, paper questionnaires were sent to the registered residential addresses of those selected. We made at least three attempts to follow-up each nonresponder. Potential participants became ineligible if we were notified that they were living overseas, were not sufficiently literate in English to complete the questionnaire or had died. The study used the core questionnaire from the GENACIS study, an international collaborative study of gender, alcohol and culture, which was designed to enable cross-national comparisons of a range of characteristics and behaviours related to alcohol consumption. ${ }^{10}$ Minor adaptations were made to the wording and layout of the questionnaire for the NZ context, and to aid self-completion. Sample size was

Table 1 Prevalence of at least one incident of victimisation or perpetration of partner aggression in the past 2 years, by gender and age group, in the whole study population*

\begin{tabular}{lccc}
\hline & $\begin{array}{c}\text { Sample } \\
\mathbf{n}(\%)\end{array}$ & \multicolumn{2}{c}{ Aggressive incident in last 2 years } \\
Age group (years) & & & Verpetration (\%) \\
$18-20$ & $52(2.7)$ & $14(26.9)$ & $14(26.9)$ \\
$21-30$ & $239(12.6)$ & $47(19.7)$ & $49(20.5)$ \\
$31-40$ & $372(19.6)$ & $72(19.4)$ & $73(19.6)$ \\
$41-50$ & $475(25.0)$ & $56(11.8)$ & $61(12.8)$ \\
$51-60$ & $443(23.3)$ & $32(7.2)$ & $44(9.9)$ \\
$61-70$ & $300(15.8)$ & $23(7.7)$ & $19(6.3)$ \\
Missing & $19(1.0)$ & $4(21.1)$ & $2(10.5)$ \\
Total & 1900 & $248(13.1)$ & $262(13.8)$ \\
\hline *Denominator of proportions includes respondents with and without partners, and includes abstainers. &
\end{tabular}


Table 2 Most aggressive act in last 2 years, by gender, whole study population* (\%)

\begin{tabular}{|c|c|c|c|c|}
\hline & \multicolumn{2}{|c|}{ Women $(n=1067)$} & \multicolumn{2}{|l|}{ Men $(n=833)$} \\
\hline & Victimisation & Perpetration & Victimisation & Perpetration \\
\hline Any aggressive act & $126(11.8)$ & $166(15.6)$ & $122(14.6)$ & $95(11.4)$ \\
\hline None & 885 (82.9) & 830 (77.8) & 669 (80.3) & $679(81.5)$ \\
\hline Missing/refused & $56(5.3)$ & $71(6.7)$ & $43(5.2)$ & $59(7.1)$ \\
\hline \multicolumn{5}{|l|}{ Most aggressive act (last 2 years) } \\
\hline Push, shove or grab & $63(5.9)$ & $63(5.9)$ & $41(5.0)$ & $59(7.1)$ \\
\hline Slap or punch & $6(0.6)$ & $35(3.3)$ & $25(3.0)$ & $14(1.7)$ \\
\hline Kicked, beat up, hit with object & $10(1.0)$ & $8(0.8)$ & $9(1.1)$ & $1(0.1)$ \\
\hline Throw something & $21(2.0)$ & $41(3.8)$ & $31(3.7)$ & $11(1.3)$ \\
\hline Threaten & $15(1.4)$ & $10(0.9)$ & $7(0.8)$ & $7(0.8)$ \\
\hline Threaten with weapon & $2(0.2)$ & 0 & $3(0.4)$ & 0 \\
\hline Other & $9(0.8)$ & $9(0.8)$ & $5(0.7)$ & $3(0.4)$ \\
\hline
\end{tabular}

based on an anticipated response rate of $60 \%$, providing 2400 participants to estimate a range of alcohol-related measures. Guidance was taken from previous use of the questionnaire in other settings.

\section{Measures}

Partner aggression (victimisation and perpetration)

Participants were asked 'What is the most physically aggressive thing done to you in the last 2 years by someone who was or had been in a close romantic relationship with you (such as wife, husband, boyfriend or girlfriend)?'

A checklist of possible responses was offered, as well as a free text field for alternative descriptions, and an option for 'No physical aggression'. This was followed by a series of questions referring to the particular incident if one had been identified. To assess the perceived impact of the aggression, respondents were asked to rate the severity of the act, how angry they were just after the incident, and how scared they were (each on a scale of $1-10$ with anchor points at the extremes).

Alcohol involvement in the identified incident was measured with the question

'Had you or the other person been drinking before this incident?'

Possible responses were 'Both,' 'You had been drinking but your partner had not,' 'Your partner had been drinking but you had not' and 'Neither.'
A parallel series of questions were asked regarding the most aggressive thing the respondent had done to someone in a close romantic relationship with him/her in the same 2-year period.

\section{Drinking patterns}

We asked respondents to indicate the frequency of days on which they drank any alcohol over the past 12 months, and the typical number of drinks they consumed per occasion. They also indicated the frequency of drinking five or more standard drinks on a single occasion ( $\geq 50 \mathrm{~g}$ ethanol). Response options were presented as tick boxes with predefined values. Heavy episodic drinking (HED) was defined as drinking $\geq 50 \mathrm{~g}$ of alcohol on a single occasion at least once a month. Respondents who were in intimate relationships were asked about their partner's frequency of drinking and usual amount consumed on an occasion by their partner.

\section{Analysis}

We excluded from our analysis any respondents who indicated that they were in a same-sex relationship (12 females, seven males), as the numbers were too small to analyse them separately. We also excluded the six respondents who had gender details missing (these respondents had very few data). Thus, there were 1900 respondents analysed.

Table 3 Involvement of alcohol in most aggressive act reported (\%)

\begin{tabular}{|c|c|c|c|c|}
\hline & \multicolumn{2}{|l|}{ Women } & \multicolumn{2}{|l|}{ Men } \\
\hline & $\begin{array}{l}\text { Victimisation, } \\
n=153\end{array}$ & $\begin{array}{l}\text { Perpetration, } \\
\mathrm{n}=159\end{array}$ & $\begin{array}{l}\text { Victimisation, } \\
n=154\end{array}$ & $\begin{array}{l}\text { Perpetration, } \\
\mathrm{n}=\mathbf{8 9}\end{array}$ \\
\hline \multicolumn{5}{|c|}{ Alcohol involvement } \\
\hline Both & $22(14.4)$ & $23(14.5)$ & $23(14.9)$ & 15 (16.9) \\
\hline $\begin{array}{l}\text { Respondent } \\
\text { only }\end{array}$ & $1(0.7)$ & $5(3.1)$ & $7(4.6)$ & $3(3.4)$ \\
\hline Partner only & $34(22.2)$ & $19(12.0)$ & $9(5.8)$ & $4(4.5)$ \\
\hline Neither & $96(62.8)$ & $112(70.4)$ & $115(74.7)$ & $67(75.3)$ \\
\hline
\end{tabular}


Aim 1

Unadjusted proportions were calculated to estimate prevalence and severity of aggression, and the proportion that involve drinking.

Aim 2

A two-way analysis of variance was used to compare the mean level of aggression, anger and fear in the most serious incident of partner aggression towards the respondent and by the respondent as a function of gender and whether drinking at the time was reported. Post-hoc pairwise comparisons were considered significant at the 0.01 level.

\section{Aim 3}

Three multinomial (generalised logit) models were fitted to investigate associations between usual drinking patterns and partner aggression, by modelling the threelevel outcome variable of aggression and drinking with the following levels: (1) no aggression, (2) aggression with no alcohol involved and (3) aggression with alcohol involved (either party was drinking or both). The first model investigated whether drinking, per se, predicted aggression to or from the partner. The second model investigated whether the amount of drinking by the respondent and partner was associated with victimisation. The third model was similar, with the outcome being the three-level variable of drinking and perpetration (no perpetration, perpetration with no drinking and perpetration with drinking). All models included age and gender of the respondent. Additionally, logistic models comparing aggression without alcohol with aggression with alcohol were fitted.

\section{RESULTS}

\section{Participants}

Usable data were received from 1925 respondents (49\% of original sample). There were 110 people found to be ineligible, and the remaining 1965 people were considered 'non-responders.' Non-responders included people who declined to participate, those whose unopened questionnaires were sent back marked 'Return to Sender' and people for whom there was no evidence of contact (one-third of the original sample). For this analysis, the exclusion of respondents in same-sex relationships and those with missing data on gender resulted in 1900 participants.

The age distribution of the study participants is shown in table 1.

Seven per cent of respondents reported that they had not drunk any alcohol in the past 12 months, and the same proportion reported that their partner was an abstainer.

\section{Experience of partner aggression}

The proportion of respondents reporting at least one incident of partner aggression in the past 2 years is described by gender and age group in table 1 . The nature of these incidents is summarised by gender in table 2. The denominator for these estimates is the study
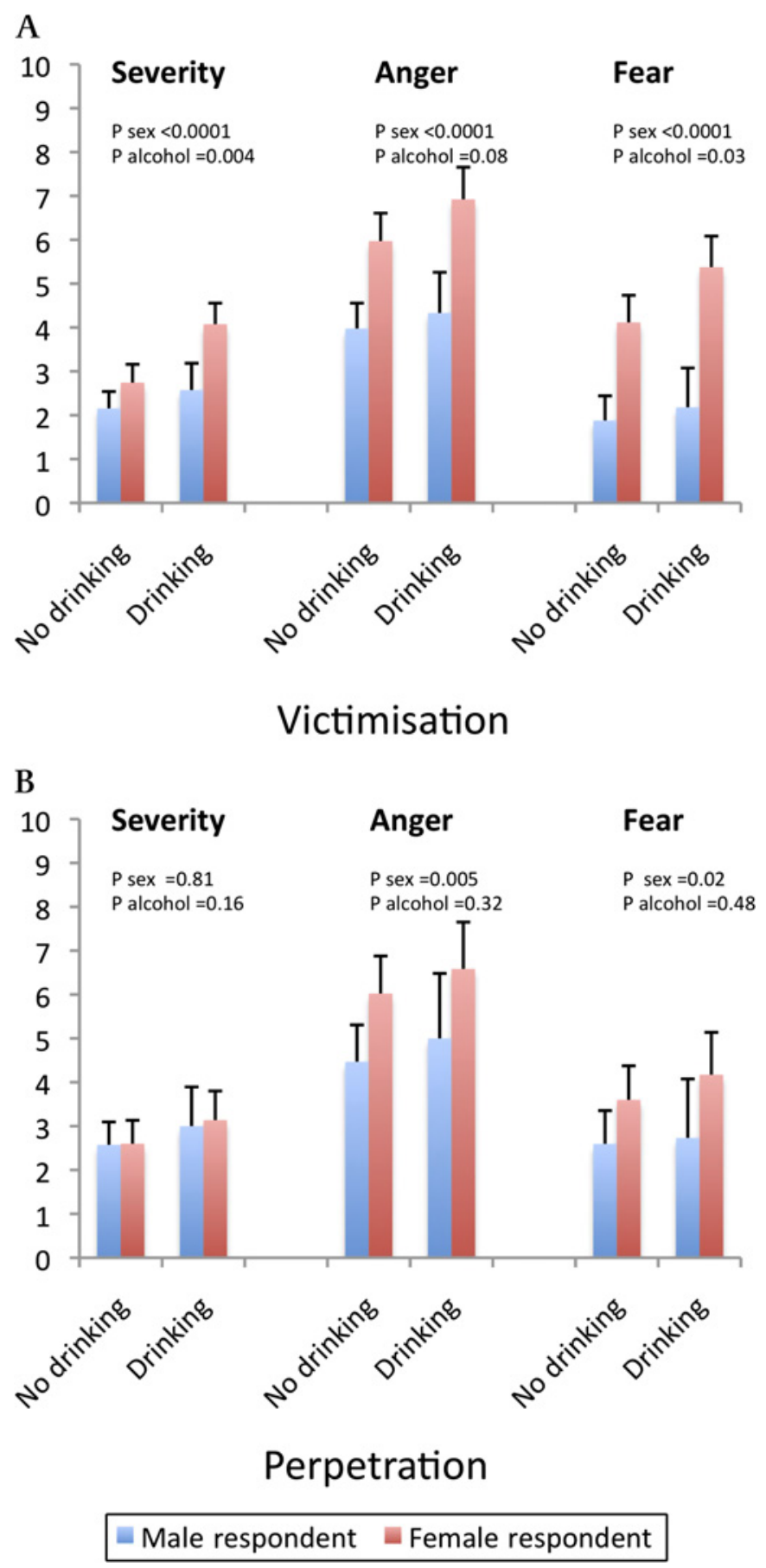

Figure 1 Self-reported severity, anger and fear associated with aggression, by role, gender and alcohol involvement: mean scores with 95\% Cls. (A) Victimisation. (B) Perpetration.

population, with and without partners and including abstainers.

Approximately $15 \%$ of men and $12 \%$ of women reported experiencing an aggressive act by a partner, and $11 \%$ of men and $16 \%$ of women reported being aggressive towards a partner in the past 2 years. A minority of these respondents $(3.6 \%$ of men and $4.5 \%$ of women) reported both. Reported aggression showed a negative gradient with age, with younger people more likely to report either victimisation or perpetration. 
Table 4 Association* of usual drinking patterns with reported victimisation by partner

\begin{tabular}{lll}
\hline & $\begin{array}{l}\text { Victimisation, no drinking } \\
\text { OR }(\mathbf{9 5 \%} \mathbf{~ C l})\end{array}$ & $\begin{array}{l}\text { Victimisation, drinking } \\
\text { OR (95\% Cl) }\end{array}$ \\
\hline Age & $0.81(0.79$ to 0.82$)$ & $0.82(0.79$ to 0.84$)$ \\
Gender & $0.52(0.33$ to 0.82$)$ & $0.78(0.38$ to 1.59$)$ \\
Heavy episodic drinking & $1.18(0.67$ to 2.10$)$ & $2.93(1.39$ to 6.17$)$ \\
Drinking frequency & $0.94(0.80$ to 1.10$)$ & $1.10(0.83$ to 1.45$)$ \\
Typical volume & $1.05(0.99$ to 1.11$)$ & $1.05(0.96$ to 1.15$)$ \\
Partner frequency & $0.90(0.79$ to 1.01$)$ & $1.45(1.10$ to 1.90$)$ \\
Partner volume & $1.03(0.97$ to 1.09$)$ & $1.11(1.04$ to 1.19$)$ \\
\hline *Multinomial model with reference category 'No aggressive act in the last 2 years.'
\end{tabular}

Table 2 shows the nature of the most aggressive act reported, and the proportion of missing responses; approximately $5 \%$ for victimisation and $7 \%$ for perpetration.

\section{Involvement of alcohol}

Among respondents who reported aggressive acts, women reported alcohol involvement more often than men, and particularly male-only drinking when they were victimised (table 3). Men reported more often than women that both partners were drinking when a man was aggressive towards a partner, and a higher proportion of incidents when the woman was the only one drinking.

\section{Severity, anger and fear}

Figure 1 shows the results of the two-way ANOVA including mean scores out of 10 for the respondent's perception of severity of aggression, level of anger just after the incident and the level of fear experienced, for men and women separately and by involvement of alcohol.

Post-hoc pairwise comparisons showed that women scored severity, anger and fear significantly higher than men when reporting victimisation. The mean scores for severity of aggression were significantly higher when drinking at the time of the incident was reported.

As perpetrators of aggression (figure 1B), men and women were much more similar in their responses. However, as the aggressor, women's anger scores were significantly higher than men's. No statistically significant interactions were found between gender and drinking at the time of the incident for either victimisation or perpetration.

\section{Association of aggressive acts with drinking patterns of} partners

Aggressive acts without drinking, and aggressive acts that involved someone drinking, were compared with no aggression in the past 2 years, using multinomial models. In the first model that included only the drinking status (current abstainer or drinker) of the respondent and their partner, as well as age and gender, we found that simply being a drinker did not predict partner aggression (data not shown). However, when we included variables characterising the drinking pattern of respondents and partners in the model, we found that drinking patterns of both partners were associated with victimisation and with perpetration of aggression in incidents where alcohol was involved.

HEDby the respondent was associated with a threefold increase in reported victimisation involving drinking (OR 2.9, 95\% CI 1.4 to 6.2), and a doubling of reported perpetration involving drinking (OR 2.2, 95\% CI 1.0 to 4.7). Partner drinking frequency and partner usual volume were also positively associated with both victimisation and perpetration that involved drinking, although less strongly (tables 4, 5).

As shown in table 5, the volume of drinking of a partner was associated with a modest increase in aggression towards that partner that did not involve drinking (OR $1.05,95 \%$ CI 1.0 to 1.1 ). This was the only drinking-pattern variable that had a statistically significant association with aggressive incidents that did not involve drinking at the time.

Interactions between gender and drinking pattern variables were examined and were not found to be statistically significant.

Table 5 Association* of usual drinking patterns with reported perpetration of partner aggression

\section{Perpetration, no drinking OR $(95 \% \mathrm{Cl})$}

Age

Gender

Heavy episodic drinking

Drinking frequency

Typical volume

Partner frequency

Partner volume
$0.82(0.81$ to 0.84$)$

1.60 (1.02 to 2.51$)$

0.75 (0.40 to 1.39$)$

$1.02(0.88$ to 1.19$)$

1.05 (0.99 to 1.12$)$

$0.82(0.73$ to 1.02$)$

1.05 (1.00 to 1.11$)$
Perpetration, drinking OR $(95 \% \mathrm{CI})$

0.87 (0.85 to 0.89$)$

1.35 (0.65 to 2.80$)$

2.16 (1.01 to 4.66$)$

$1.21(0.91$ to 1.62$)$

1.06 (0.97 to 1.17$)$

$1.39(1.05$ to 1.83$)$

$1.12(1.05$ to 1.20$)$

*Multinomial model with reference category 'No aggressive act in the last 2 years. 
Table 6 Association* of heavy episodic drinking with alcohol involvement in reported partner aggression

\begin{tabular}{llr}
\hline & $\begin{array}{l}\text { Victimisation } \\
\text { OR (95\% Cl) }\end{array}$ & $\begin{array}{l}\text { Perpetration } \\
\text { OR (95\% Cl) }\end{array}$ \\
\hline Age & $1.03(1.00$ to 1.06$)$ & $1.04(1.01$ to 1.07$)$ \\
Gender & $1.56(0.74$ to 3.29$)$ & $0.96(0.44$ to 2.11$)$ \\
Heavy episodic drinking & $3.02(1.43$ to 6.42$)$ & $3.31(1.45$ to 7.57$)$ \\
Heavy episodic drinking partner & $2.50(1.03$ to 6.04$)$ & $4.05(1.73$ to 9.48$)$ \\
\hline "Logistic regression model with reference category aggression not involving drinking by either partner.
\end{tabular}

Table 6 presents the results of the logistic regression model of the associations of HED by each partner with the occurrence of aggression involving alcohol, compared with aggression not involving alcohol. There was a strong association of HED with the involvement of alcohol in the aggression reported, for each partner and both victimisation and perpetration.

\section{DISCUSSION}

This survey found that rates of victimisation and perpetration of aggression reported by men and women were similar, with slightly more women reporting being aggressive and slightly more men reporting victimisation. Alcohol was involved in over a quarter of incidents, and women reported alcohol involvement more often than men, particularly male-only drinking when they were the victim. Women also reported higher levels of severity, anger and fear associated with victimisation, and drinking at the time of the incident was associated with higher severity scores for reported victimisation. When they were the aggressors, women's responses were more similar to men's. HED by respondents was associated with a threefold increase in victimisation involving alcohol, and doubling of reported perpetration of aggression involving alcohol. Usual drinking patterns were not associated with aggressive acts that did not involve drinking at the time. HED by either partner was associated with an increase in the risk that alcohol would be involved in any reported incident.

Some limitations of this study need acknowledgement. The modest response rate limited power and, since selfselection into the study could plausibly be related to some of the variables of interest, could have resulted in bias. Experience from previous alcohol survey research and analysis of non-response patterns in these survey data $^{17} 18$ suggest that heavy drinkers, the youngest age group, and men will be under-represented in these findings. In addition, there is under-representation of young people on the electoral roll compared with the general population, although approximately $92 \%$ of the eligible population is enrolled. Therefore, we expect that the prevalence of partner aggression will be underestimated. The associations seen between drinking variables and aggression are probably less affected by response bias, as they are less reliant on the representativeness of the sample. The sensitivity of the questions may have also led to under-reporting among respondents, and this may have varied by gender. Analysis of the data in further detail was constrained by both sample size and the low prevalence of some scenarios. In particular, only one female respondent reported that the most serious act of aggression towards her partner in the last 2 years occurred when she was the only one drinking.

When interpreting these findings, one needs to bear in mind that the study did not collect reports from male and female partners of the same couple, and response biases may affect the gender comparisons. As well as this, there may be a gender difference in the reporting of incidents of aggression if the 'most aggressive thing' was of relatively minor severity. The inclusion of minor aggression was important because of the association with subsequent escalation of violence. ${ }^{19}$ Although these findings represent the experience of a representative sample of couples from the general population, rather than those selected for a history of violence or abuse, it has been observed that men and women in abusive relationships tend to minimise men's violence and remember women's violence. ${ }^{20}$ This may also affect these results.

Previous NZ studies have estimated the occurrence of intimate-partner violence in a range of population groups, over different time periods, and using a different threshold of aggression, none of which are directly comparable with this study. ${ }^{13}$ We are not aware of previous NZ research that has investigated the role of alcohol and gender in incidents of aggression.

The GENACIS questions about partner aggression have been used in surveys in other countries with which NZ commonly compares itself: Australia, Canada and UK. The Australian survey ${ }^{21}$ was conducted in the state of Victoria in the same year as this study (2007). The proportion of the population reporting any partner aggression in the previous 2 years was substantially lower than in the study reported here $(8 \%$ of women and $9 \%$ of men reported victimisation; $6 \%$ of both women and men reported perpetration), but the proportion of those reporting alcohol involvement in the aggression was higher. Comparisons are limited by the modest representativeness of both surveys (49\% response in NZ and cooperation rate of $38 \%$ in Australia).

In Canada, the GENACIS questions have been used with a much larger representative sample $(>14000)$ in 2004-2005. The pattern of findings was similar to those of the NZ study although the 2-year prevalence of aggression was lower, being more similar to the 
Australian study. ${ }^{19}$ In the UK survey conducted in 2000 , the prevalence of partner aggression was slightly higher than in the current NZ study, showing the same pattern of men reporting more victimisation than women and women reporting more perpetration. ${ }^{4}$ The involvement of alcohol in aggressive incidents was also slightly higher than in NZ (35-40\% of incidents).

Consistent with the NZ findings, both the Canadian and UK studies demonstrated that the drinking pattern of the respondent and their partner predicted involvement in aggression involving alcohol but not aggression that did not involve alcohol.

More recently a cross-national comparison of 13 diverse countries has been published, ${ }^{8}$ which found that a greater severity of aggression was consistently reported by women than by men. The severity of aggression was also associated with alcohol use at the time of the incident in virtually all countries studied, despite the large cultural differences with regard to alcohol and to aggression. There was no significant interaction between alcohol involvement and gender in this analysis, suggesting that the association between alcohol involvement and severity of aggression does not vary greatly between men and women. These findings, consistent with the NZ results reported here, support the hypothesis of a direct contribution of alcohol use to aggression severity through its pharmacological effects on the perpetrator, the victim or both, rather than primarily through cultural mechanisms.

This hypothesis is further supported by the research of Wells $e t a l,{ }^{22}$ which showed that the level of intoxication, rather than alcohol involvement per se, was important in the escalation of violence and that the number of drinks consumed in the $6 \mathrm{~h}$ before an aggressive incident was associated with the usual pattern of drinking of the perpetrator. This suggests that the association of usual drinking pattern with alcohol-involved violence is at least partly mediated by higher levels of intoxication in heavy drinkers, rather than by heavy drinkers being more aggressive in general.

Any causal relationship between alcohol consumption and the prevalence and severity of partner aggression will be of particular public-health importance in countries where HED is widely accepted, such as New Zealand. Efforts to reduce harm need to include both individual-level interventions such as more intensive screening for partner violence when one or both partners are heavy drinkers, and population-level interventions to reduce the frequency and magnitude of heavy drinking in the population.

\section{CONCLUSION}

The findings from this study suggest that the experience of intimate-partner aggression in a cross-section of households differs considerably by gender and the involvement of alcohol, and that 'counts' of aggressive acts in a population-based survey do not reflect the reality of gender differences. The involvement of alcohol in an incident of aggression is associated with increased severity, suggesting that alcohol is escalating aggression or affecting the perception of the aggressive act by the victim, or both. An HED pattern is associated with increased victimisation as well as perpetration of partner aggression in which alcohol is involved.

Acknowledgements The questionnaire was developed by the members of the International Research Group on Gender and Alcohol (IRGGA) as part of the international GENACIS collaboration (Gender, Alcohol and Culture: an International Study) (S Wilsnack, PI).

Funding The NZ Gender, Alcohol, and Culture International Study survey was funded by an internal research grant to JC from the University of Otago (UORG 2007).

\section{Competing interests None.}

Ethical approval Ethics approval was provided by the University of Otago Human Ethics Committee (06/171).

Contributors JLC and KK conceived and designed the study, obtained funding, supervised data collection, interpreted the findings and wrote the paper. MLB designed and conducted the data analysis and contributed to the interpretation and writing of the paper. KC collected and prepared the data, and contributed to the interpretation and writing of the paper. All authors approved the final version of the article.

Provenance and peer review Not commissioned; externally peer reviewed.

Data sharing statement No additional data available.

\section{REFERENCES}

1. Babor T, Caetano R, Casswell S, et al. Alcohol: No Ordinary Commodity - Research and Public Policy. Oxford: Oxford University Press, 2003.

2. Connor J, You R, Casswell S. Alcohol-related harm to others: a survey of physical and sexual assault in New Zealand. $N Z$ Med J 2009;122:10-20.

3. Stevenson R. National Alcohol Assessment. Wellington: New Zealand Police, 2009.

4. Graham K, Plant M, Plant M. Alcohol, gender and partner aggression: a general population study of British adults. Addict Res Theory 2004;12:385-401.

5. Leonard KE, Quigley BM. Drinking and marital aggression in newlyweds: an event-based analysis of drinking and the occurrence of husband marital aggression. J Stud Alcohol 1999;60:537-45.

6. Testa M, Quigley BM, Leonard KE. Does alcohol make a difference? Within-participants comparison of incidents of partner violence. $J$ Interpers Violence 2003;18:735-43.

7. Kaufman-Kantor G, Asdigian N. When women are under the influence: does drinking or drug use by women provoke beatings by men? In: Galanter M, ed. Recent Developments in Alcoholism. New York: Plenum Press 1997:315-36.

8. Graham K, Bernards S, Wilsnack S, et al. Alcohol may not cause partner violence but seems to make it worse: A cross national comparison of the relationship between alcohol and severity of partner violence. J Interpers Violence 2011;26:1503-23.

9. Giancola PR, Helton EL, Osborne AB, et al. The effects of alcohol and provocation on aggressive behavior in men and women. J Stud Alcohol 2002;63:64-73.

10. Wilsnack R, Wilsnack S, Kristjanson A, et al. Gender and alcohol consumption: patterns from the multinational GENACIS project. Addiction 2009;104:1487-500.

11. Graham K, Wells S. The two worlds of aggression for men and women. Sex Roles 2001;45:595-622.

12. Straus MA, Hamby SL, BoneyMcCoy S, et al. The revised Conflict Tactics Scales (CTS2)-Development and preliminary psychometric data. J Fam Issues 1996;17:283-316.

13. Fanslow J. Beyond Zero Tolerance: Key Issues and Future Directions for Family Violence Work in New Zealand. Wellington: Families Commission, 2005.

14. Giles J. 'Woman bites dog'-making sense of media and research reports that claim women and men are equally violent. $N Z$ Med J 2005;118:U1731.

15. Leonard KE, Quigley BM, Collins RL. Physical aggression in the lives of young adults-Prevalence, location, and severity among college and community samples. J Interpers Violence 2002;17:533-50. 
16. Gondolf EW, Beeman AK. Women's accounts of domestic violence versus tactics-based outcome categories. Violence against Women 2003;9:278-301.

17. Connor JL, Kypri K, Bell ML, et al. Alcohol outlet density, levels of drinking and alcohol-related harm in New Zealand: a national study. $J$ Epidemiol Community Health. Published Online First: 14 October 2010. doi:10.1136/jech.2009.104935.

18. Meiklejohn J, Connor J, Kypri K. Low survey response rates and the effect on estimates of drinking in a general population sample [abstract]. AEA Annual Scientific Meeting 2010. Sydney: Australasian Epidemiologist, 2010:126.

19. Graham K, Bernards S. Canada: alcohol and partner physical aggression in the 10 provinces. In: Graham K, Bernards S, Munne M, et al, eds. Unhappy Hours: Alcohol and Partner Aggression in the Americas. Washington DC, WA, USA: PAHO, 2008.

20. Dobash R, Dobash R, Cavanagh K. Separate and intersecting realities: A comparison of men's and women's accounts of violence against women. Violence against Women 1998:4:382-414.

21. Matthews S. The Social Location of Intimate Partner Aggression and the Association with Alcohol Consumption. 34th Annual Alcohol Epidemiology Symposium of the Kettil Bruun Society. Victoria, BC: KBS, 2008.

22. Wells S, Graham K. Aggression involving alcohol: realtionship to drinking patterns and social context. Addiction 2003; $98: 33-42$. 